\title{
AN APPLICATION OF PERTURBATION ANALYSIS TO A REPLACEMENT PROBLEM IN MAINTENANCE THEORY
}

\author{
Michael C. Fu \\ College of Business and Management \\ University of Maryland at College Park \\ College Park, Maryland $207+2$
}

\author{
Jian-Qiang Hu \\ Department of Manufacturing Engineering \\ Boston University \\ Boston. Massachusetts 02215
}

\author{
Leyuan Shi \\ Institute for Advanced Computer Studies \\ University of Maryland at College Park \\ College Park, Maryland 20742
}

\begin{abstract}
We consider the problem of sensitivity analysis for replacement problems in maintenance theory. In particular, we apply the technique of perturbation analysis to derive estimators for the gradient of a cost performance measure with respect to replacement parameters for a variation of a problem in L'Ecuyer (1990).
\end{abstract}

\section{INTRODUCTION}

We consider a replacement problem which arises in maintenance/reliability theory for the following system of components with random lifetimes:

- the system comprises $N$ identical components;

- the components are independent of each other;

- component service times are random variables with increasing (non-decreasing) failure rates and identically distributed p.d.f. $f$ and c.d.f. $F$;

- replacements are made immediately upon failure and possibly preventively;

- replacements are instantaneous.

The following cost parameters define the problem:

$$
\begin{aligned}
c_{I} & =\text { cost incurred for an intervention, } \\
c_{R} & =\text { cost per component replaced, }
\end{aligned}
$$

where $c_{I}$ can be thought as a fixed cost per intervention, $c_{R}$ as a per-unit replacement cost, and often (but not always) $c_{I}>>c_{R}$. The problem is to determine a preventive maintenance policy to minimize the longrun average cost. We will use the term replacement epoch to refer to an epoch in which intervention for replacement occurs. A maintenance policy will govern the timing of replacement epochs and the number of components replaced at a particular epoch.
We will consider a performance measure given by

$$
\begin{aligned}
J= & \lim _{n \rightarrow \infty} \bar{L}_{n}, \quad \bar{L}_{n}=L_{n} / n, \\
L_{n}= & \sum_{i=1}^{n} g_{i}, \quad g_{i}=c_{I}+c_{R} R_{i}, \\
R_{i}= & \# \text { components replaced at } \\
& \text { the ith replacement epoch, } \\
n= & \# \text { replacement epochs. }
\end{aligned}
$$

We note that the usual time-average performance measure can also be handled by expressing it as

$$
\frac{L_{n(T)}}{T} \approx\left(\frac{L_{n}}{n}\right)\left(\frac{n}{T(n)}\right),
$$

where $n(T)$ is the number of replacement epochs over $[0, T]$ and $T(n)$ is the $n$th replacement epoch. For the gradient estimation problem, the chain rule can be applied to the two terms, resulting in two separate gradients which can be estimated.

We will assume that the distribution for the tentative service time of each component (i.e., how long the component would be left in service if there were not simultaneous replacements) contains a controllable parameter $\theta_{1}$. For example, $\theta_{1}$ could be the maximum amount of time a component is allowed to remain in the system. Furthermore, we will consider simultaneous replacements done under the following (not necessarily optimal) class of threshold policies parameterized by $\theta_{2}$ :

( $\pi)$ Whenever a component fails, all components older than age $\theta_{2}$ are replaced.

For example, the replacement of tools in a flexible manufacturing system often follows a maintenance policy of this type, since each intervention often involves a major interruption of the system. Thus, the optimal setting of the parameters $\theta_{1}$ and $\theta_{2}$ are of primary importance. Gradient estimates can be very useful in determining these settings (see, e.g., 
Fu 1994), and the goal of this paper is to provide an unbiased estimator for $d J / d \theta, \theta=\left(\theta_{1}, \theta_{2}\right)$ under policy $\pi$. A slightly different version of this problem was presented in L'Ecuyer (1990) as an open problem for gradient estimation procedures. There, $\theta_{1}$ was taken as another threshold parameter characterizing the following policy:

$\left(\boldsymbol{\pi}^{\prime}\right)$ Whenever a component fails or reaches age $\theta_{1}$, all components older than age $\theta_{2}$ are replaced.

Our problem subsumes this by taking $\theta_{1}$ as an upper bound on the service time of a component, except that by using this modeling device we cannot then distinguish between interventions costs due to $\theta_{1}$ replacements versus those due to failures.

Aside from finite difference estimates, there are chiefly two techniques available to estimate the gradient: the likelihood ratio (also known as the score function) method and perturbation analysis. As noted by L'Ecuyer (1990), the likelihood ratio method is often ill-equipped to handle threshold types of parameters, although Rubinstein (1992) proposes one way to overcome some inherent difficulties. On the other hand, perturbation analysis can often be applied to these types of problems; some examples include Gong (1988), Wardi et al. (1991), Caramanis and Liberopolous (1992), and Fu (1993). In this paper, we apply the technique of smoothed perturbation analysis (SPA) to derive gradient estimators for the finite-horizon replacement problem described above. We then simplify the estimators for the long-run performance measure.

\section{THE ESTIMATOR}

The technique of smoothed perturbation analysis (SPA) often works for cases where the technique of infinitesimal perturbation analysis (IPA) fails. For our derivative estimation problem, IPA gives zero for both parameters, which is obviously incorrect, so we apply SPA. The intuitive idea behind SPA is that by conditioning on certain sample path quantities, it may be possible to "smooth" the performance measure such that a sample path derivative will then be unbiased; see, e.g., Gong and Ho (1987), Glasserman (1991), Ho and Cao (1991). Here, we will derive our estimators using the ideas introduced in $\mathrm{Fu}$ and $\mathrm{Hu}$ (1992). In particular, the main ideas are the following:

- Identify critical "events" with respect to the performance measure and parameter of interest;

- Select conditioning quantities to allow a calculation/estimation of the expected rate of change of these critical events due to a perturbation and the resulting effect on the performance measure;

- Construct a degenerative nominal path (DNP) and a perturbed path (PP) to represent the effect, and find efficient ways to estimate DNP and PP from the original sample path.

Before we begin the analysis, let us describe how the actual simulation would take place, which intuitively is simple enough, but does not fall as naturally under the usual Generalized Semi-Markov Process (GSMP) framework as most queueing systems. As in the GSMP framework, we assign clocks, but instead of associating each event with a clock, we associate with each component a clock, $C_{j}, j=1, \ldots, N$, which tentatively will give the time until the next replacement, with the allowance of "preemptive" replacements due to our policy. Let $Y_{j} \sim F\left(\theta_{1}\right)$ i.i.d.

INITIALIZE: (assume all components new)

$$
C_{j}:=Y_{j} \sim F\left(\theta_{1}\right), j=1, \ldots, N .
$$

\section{TIMING MECHANISM:}

Next replacement epoch occurs at $\tau$ from the present, where $\tau=\min _{j \in\{1, \ldots, N\}} C_{j}$ (ties broken arbitrarily). REPLACE:

- the component generating the epoch, i.e., $\arg \min _{j \in\{1, \ldots, N\}} C_{j}$

- any other component with age $>\theta_{2}$.

\section{UPDATE CLOCKS:}

- For each replaced component, generate new clock time

$$
C_{j} \sim F
$$

- For each non-replaced component, $C_{j}:=C_{j}-\tau$.

At each intervention epoch, on the sample path for each component, one of the following "events" occurs:

$$
\begin{aligned}
& \alpha=\text { forced intervention replacement } \\
& \gamma=\text { early preventive replacement } \\
& \phi=\text { null event, i.e., no replacement. }
\end{aligned}
$$

It is clear that $\gamma$ can only occur for one component in conjunction with $\alpha$ occurring at another component. Thus, $\alpha$ generates the replacement epoch, at which time either $\gamma$ or $\phi$ occurs at each of the other components. It is the interaction of these events among components as a function of $\theta_{1}$ and $\theta_{2}$ which determines the gradient estimator. 
We define

$$
\begin{aligned}
e_{j}(t)= & \text { event at component } j \text { at epoch } t, \\
Y_{j}(t)= & \text { service time of component } j \\
& \text { in service at epoch } t \\
\nu_{j}(t)= & \text { age of component } j \\
& \text { in service at epoch } t, \\
\eta_{j}(t)= & \text { residual service time of component } j \\
& \text { in service at epoch } t \\
t_{i}= & \text { the } i \text { th replacement epoch, } \\
\tau_{i+1}= & t_{i+1}-t_{i}, \text { time between the } \\
& i \text { th and }(i+1) \text { st replacement epoch. }
\end{aligned}
$$

Note that the "service time" is not really the true service time, since early replacement can occur according to $\theta_{2}$.

Intuitively, the estimator is the sum of the following types of terms:

$$
(\text { EXCHANGE RATE })_{i}\left(\bar{L}_{n}^{P P(i)}-\bar{L}_{n}^{D N P(i)}\right) .
$$

The first term is a probability rate of exchange of critical events conditioned at the $i$ th replacement epoch, whereas the second term is the expected effect of this exchange. For example, in our system, one exchange we will consider is from $\gamma$ to $\phi$ at a component: the immediate effect is a reduction in cost of $c_{R}$, but there are further effects, since in the former case, a component is replaced and in the latter, an old one is left in. The latter case is the $P P$ sample path (under a certain limit) and the former is the $D N P$ sample path (under a certain limit).

To shorten notation a bit, we introduce the superscripted " $i$ " to indicate quantities at the $i$ th replacement epoch $t_{i}^{+}$(assume right continuity). We will use the subscript "** to indicate the index of the component which generates the next replacement epoch, e.g., $\nu_{*}^{i}$ indicates the age of the service time at $t_{i}$ of the component which will generate the $(i+1)$ st replacement epoch, and $e_{j}^{i+1}$ indicates the event occurring at component $j$ at time $t_{j+1}$. We also introduce

$$
\begin{aligned}
\xi_{i} & =\max \left\{\theta_{2}-\nu_{j}^{i}: e_{j}^{i+1}=\gamma\right\}(0 \text { if empty }), \\
\eta_{i} & =\min \left\{\min \left(\theta_{2}, Y_{j}^{i}\right)-\nu_{j}^{i}: e_{j}^{i+1}=\phi, Y_{j}^{i}-\nu_{j}^{i}: e_{j}^{i+1}=\gamma\right\} .
\end{aligned}
$$

Figure 1 depicts the three possible situations for a component $j \neq *$. In the first situation, an early replacement $(\gamma)$ occurs at $t_{i+1}$, because the component has already exceeded $\theta_{2}$ by that epoch, whereas in the second and third situations, the component is still too young $(\phi)$. The difference between the second and third situations comes in their service times; in the third situation, the component will fail before it ever reaches $\theta_{2}$, whereas in the second situation, there is a possibility that early replacement will occur before failure. The quantity $\xi_{i}$ is taken over components in the first situation, whereas $\eta_{i}$ is taken over all components. Essentially, $\xi_{i}$ is the time from $t_{i}$ that a component last exceeded the threshold $\theta_{2}$ (reached the "qualification" age for early replacement) prior to $t_{i+1}$, whereas $\eta_{i}$ is the time from $t_{i}$ that a component is first scheduled to either exceed $\theta_{2}$ or fail following the replacement epoch $t_{i+1}$. We will use a subscripted "-" to indicate the index of the component corresponding to $\xi_{i}$ (if any) and "+" to indicate the index of the component corresponding to $\eta_{i}$; from the definitions, there will always be a "+" component but not necessarily a "-" component. It is also possible that the same component be both the "+" and "-" if it corresponds to the first situation of Figure 1. Under our conditioning, $\xi_{i}$ and $\eta_{i}$ will provide lower and upper bounds, respectively, for the random variable $\tau_{i+1}$, the time to the next replacement epoch given by

$$
\tau_{i+1}=Y_{*}^{i}-\nu_{*}^{i} \text {. }
$$

The quantities used in the conditioning arguments are depicted in Figure 2.

As presented in Fu and $\mathrm{Hu}$ (1992), both right-hand and left-hand gradient estimators are available in general. For our problem, we will consider only the righthand gradient, since it can be shown that the two are equal in expectation. A summary of analysis is as follows:

$\Delta \theta_{1}>0$

- accumulated perturbations may cause an intervention to occur relatively earlier such that no event occurs $(\phi)$ for a component where there was an early preventive intervention $(\gamma)$ before; e.g., if component $j$ was originally in the first situation of Figure 1, perturbations could cause $t_{i+1}$ to occur earlier (relative to $j$ ) such that the timing at $j$ becomes that of the second situation;

- conversely, accumulated perturbations may cause an intervention to occur relatively later such that

(a) an early preventive replacement $(\gamma)$ occurs for a component where there was no event $(\phi)$ before, preempting the original replacement epoch, e.g., going from the second situation in Figure 1 to the first; or

(b) another component generates the replacement epoch $(\alpha)$, arising from the first or third situation shown in Figure 1; 

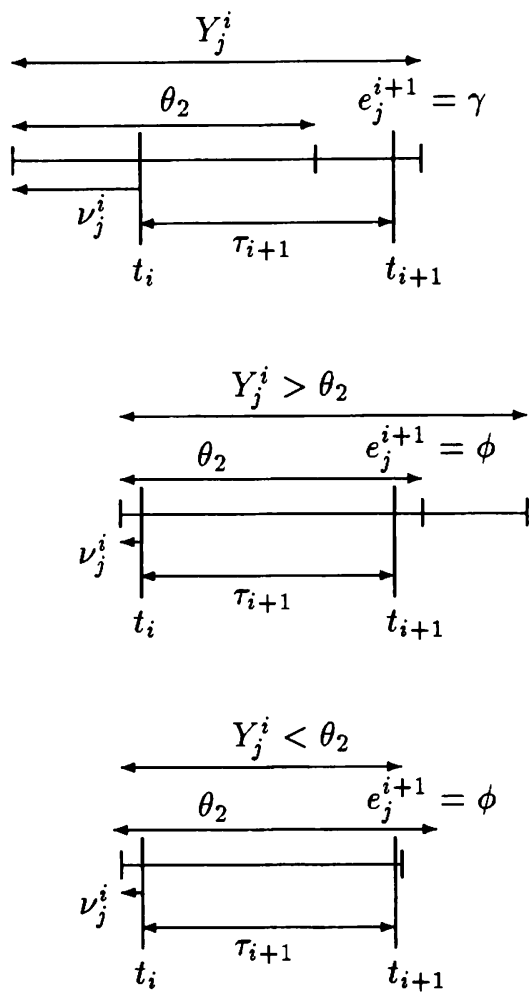

Figure 1:. Three possible situations for a component $j \neq *$.

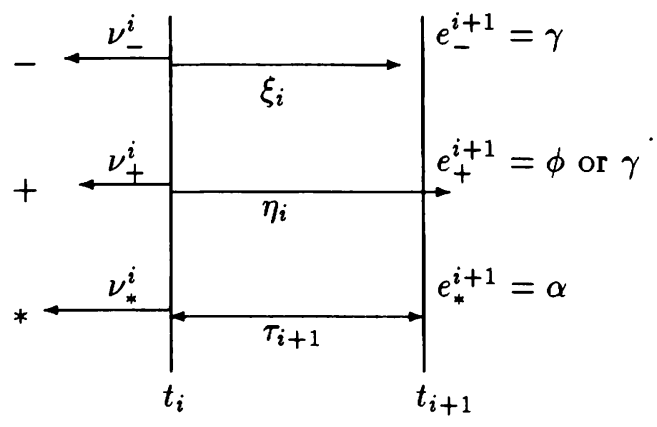

Figure 2: Quantities Used in Conditioning.
$\underline{\Delta \theta_{2}>0}$

- the increase in $\theta_{2}$ may cause an early preventive intervention $(\gamma)$ to be eliminated at a component.

The possibilities discussed above translate into the following five cases to consider:

$$
\begin{aligned}
& e_{-}^{i+1}\left[\theta_{1}\right]=\gamma, e_{-}^{i+1}\left[\theta_{1}+\Delta \theta_{1}\right]=\phi ; \\
& e_{+}^{i+1}\left[\theta_{1}\right]=\phi, e_{+}^{i+1}\left[\theta_{1}+\Delta \theta_{1}\right]=\gamma, Y_{+}^{i}>\theta_{2} \\
& e_{+}^{i+1}\left[\theta_{1}\right]=\gamma, e_{+}^{i+1}\left[\theta_{1}+\Delta \theta_{1}\right]=\alpha, Y_{+}^{i}>\theta_{2} \\
& e_{+}^{i+1}\left[\theta_{1}\right]=\phi, e_{+}^{i+1}\left[\theta_{1}+\Delta \theta_{1}\right]=\alpha, Y_{+}^{i} \leq \theta_{2} \\
& e_{-}^{i+1}\left[\theta_{2}\right]=\gamma, e_{-}^{i+1}\left[\theta_{2}+\Delta \theta_{2}\right]=\phi ;
\end{aligned}
$$

where [.] is used to indicate the dependence on the parameter value under consideration. For example, the last expression means that for the nominal value of the parameter $\theta_{2}$, there was an early preventive replacement $(\gamma)$ at the component "-" at the $(i+1)$ st replacement epoch, whereas with perturbation $\Delta \theta_{2}$, there was no such replacement $(\phi)$.

At each replacement epoch $t_{i}$, we condition on basically everything except the actual value $Y_{*}^{i}$, which determines $t_{i+1}$ through $\tau_{i+1}$, the distribution of which we wish to derive. In particular, at $t_{i}$, we condition on

$$
\left\{v_{j}^{i}, e_{j}^{i+1}, j=1, \ldots, N\right\},\left\{Y_{j}^{i}, j \neq *\right\}, \mathbf{I}\left\{Y_{*}^{i}>\theta_{2}\right\},
$$

(where $\mathbf{I}$ is the indicator function) i.e., we condition on the age of the component generating the next replacement epoch, the ages and residual service times of all other components, and all the events at $t_{i+1}$. Then, the only random variable left is the residual service time of the component generating the next replacement epoch, $\tau_{i+1}$, whose conditional distribution, given $\xi_{i}<\tau_{i+1} \leq \eta_{i}$, we now derive:

$$
\begin{aligned}
& P\left(\tau_{i+1} \leq x \mid Y_{*}^{i}>\nu_{*}^{i}, \xi_{i}<\tau_{i+1} \leq \eta_{i}\right) \\
= & P\left(Y_{*}^{i}-\nu_{*}^{i} \leq x \mid Y_{*}^{i}>\nu_{*}^{i}, \xi_{i}<Y_{*}^{i}-\nu_{*}^{i} \leq \eta_{i}\right) \\
= & \frac{P\left(\nu_{*}^{i}+\xi_{i}<Y_{*}^{i} \leq \nu_{*}^{i}+\min \left(x, \eta_{i}\right)\right)}{P\left(\nu_{*}^{i}+\xi_{i}<Y_{*}^{i} \leq \nu_{*}^{i}+\eta_{i}\right)}
\end{aligned}
$$

Thus, the conditional distribution is

$$
F_{\tau}(x)=\left\{\begin{array}{ll}
0 & \text { if } x<\xi_{i} \\
\frac{F\left(\nu_{i}^{i}+x\right)-F\left(\nu_{i}^{i}+\xi_{i}\right)}{F\left(\nu !+\eta_{i}\right)-F\left(\nu_{i}^{i}+\xi_{i}\right)} & \text { if } \xi_{i} \leq x \leq \eta_{i} \\
1 & \text { if } x>\eta_{i}
\end{array},\right.
$$

with conditional density

$$
f_{\tau}(x)= \begin{cases}\frac{f\left(\nu_{\bullet}^{i}+x\right)}{F\left(\nu_{\bullet}^{i}+\eta_{i}\right)-F\left(\nu_{\bullet}^{i}+\xi_{i}\right)} & \text { if } \xi_{i} \leq x \leq \eta_{i} \\ 0 & \text { otherwise }\end{cases}
$$


where for notational brevity, we take $\tau \equiv \tau_{i+1}$.

We first consider the easier $\Delta \theta_{2}>0$ case, for which the condition $e_{-}^{i+1}\left[\theta_{2}\right]=\gamma, e_{-}^{i+1}\left[\theta_{2}+\Delta \theta_{2}\right]=\phi$ translates into the probability (see Figure 2)

$$
\begin{gathered}
P\left(\nu_{-}^{i+1} \leq \theta_{2}+\Delta \theta_{2}, \nu_{-}^{i+1}>\theta_{2}\right) \\
=P\left(\nu_{-}^{i}+\tau \leq \theta_{2}+\Delta \theta_{2}, \nu_{-}^{i}+\tau>\theta_{2}\right),
\end{gathered}
$$

for which the appropriate rate is given by

$$
\begin{aligned}
& \lim _{\Delta \theta_{2} \rightarrow 0} \frac{P\left(\nu_{-}^{i}+\tau \leq \theta_{2}+\Delta \theta_{2} \mid \nu_{-}^{i}+\tau>\theta_{2}\right)}{\Delta \theta_{2}} \\
& = \begin{cases}\frac{f\left(\nu_{-}^{i}+\theta_{2}-\nu_{-}^{\prime}\right)}{F\left(\nu !+\eta_{i}\right)-F\left(\nu !+\theta_{2}-\nu_{-}^{i}\right)} & \text { if } \xi_{i} \leq \theta_{2}-\nu_{-}^{i} \leq \eta_{i} \\
0 & \text { otherwise }\end{cases}
\end{aligned}
$$

Now, we consider the four $\Delta \theta_{1}$ cases. Unlike the case just considered, where only the type of event was affected, and not the actual timing of replacement epochs, here there can be "propagation" of perturbations, because $\theta_{1}$ actually affects the interevent times, as well as the type of events. A perturbation in $\theta_{1}$ is propagated throughout the timing of the system according to the following rules:

\section{PROPAGATION RULES}

- $\frac{d T_{j}^{1}}{d \theta_{1}}:=\frac{d T_{j}^{i-1}}{d \theta_{1}}+\frac{d Y_{j}^{1-1}}{d \theta_{1}}$ if $e_{j}^{i}=\alpha$;

- $\frac{d T_{j}^{i}}{d \theta_{1}}:=\frac{d T_{\dot{*}}^{i}}{d \theta_{1}}$ if $e_{j}^{i}=\gamma$;

- reset all $\frac{d T_{j}^{i}}{d \theta_{1}}:=0$ at regenerative point (when $e_{j}^{i} \neq \phi \forall j$ );

where $d T_{j}^{i} / d \theta_{1}$ is a variable introduced to track the (relative) perturbations in timing at component $j$ (at epoch $t_{i}^{+}$). The first rule implements the propagation of a perturbation generated in the service time which generates the $i$ th replacement epoch. The second rule implements the propagation of the generating component to all other components which are also replaced at the $i$ th replacement epoch. The last rule is implemented because at a regenerative point, the second rule implies that every component will get the same perturbation, and hence all differences are 0 ; for our purposes the difference is all that matters, so without loss of generality, we can reset all counters to 0 .

We first define

$$
\frac{d \delta_{j}^{i}}{d \theta_{1}}=\left(\frac{d T_{j}^{i}}{d \theta_{1}}+\frac{d Y_{j}^{i}}{d \theta_{1}} \mathbf{I}_{i}\right)-\left(\frac{d T_{*}^{i}}{d \theta_{1}}+\frac{d Y_{*}^{i}}{d \theta_{1}}\right),
$$

where we define $\mathbf{I}_{i}=0$ for the first two $\Delta \theta_{1}$ cases, and $\mathbf{I}_{i}=1$ for the last two $\Delta \theta_{1}$ cases, with $\Delta \delta_{j}^{i}$ defined as the corresponding finite perturbation. Thus, this indicator function corresponds to the first and third situations in Figure 1 when there is the possibility of a switch in the component which generates the $(i+1)$ th replacement epoch.

We translate the possibilities for $\Delta \theta_{1}$ discussed above into the following four probabilities:

$$
\begin{gathered}
e_{-}^{i+1}\left[\theta_{1}\right]=\gamma, e_{-}^{i+1}\left[\theta_{1}+\Delta \theta_{1}\right]=\phi: \\
P\left(\nu_{-}^{i}+\tau-\Delta \delta_{-}^{i} \leq \theta_{2} \mid \nu_{-}^{i}+\tau>\theta_{2},\right. \\
\left.\Delta \delta_{-}^{i}>0\right) ; \\
e_{+}^{i+1}\left[\theta_{1}\right]=\phi, e_{+}^{i+1}\left[\theta_{1}+\Delta \theta_{1}\right]=\gamma, Y_{+}^{i}>\theta_{2}: \\
P\left(\nu_{+}^{i}+\tau-\Delta \delta_{+}^{i}>\theta_{2} \mid \nu_{+}^{i}+\tau \leq \theta_{2},\right. \\
\left.\Delta \delta_{+}^{i}<0, Y_{+}^{i}>\theta_{2}\right) ; \\
e_{+}^{i+1}\left[\theta_{1}\right]=\gamma, e_{+}^{i+1}\left[\theta_{1}+\Delta \theta_{1}\right]=\alpha, Y_{+}^{i}>\theta_{2}: \\
P\left(\nu_{+}^{i}+\tau-\Delta \delta_{+}^{i}>\theta_{2} \mid \nu_{+}^{i}+\tau \leq \theta_{2},\right. \\
\left.\Delta \delta_{+}^{i}<0, Y_{+}^{i}>\theta_{2}\right) ; \\
e_{+}^{i+1}\left[\theta_{1}\right]=\phi, e_{+}^{i+1}\left[\theta_{1}+\Delta \theta_{1}\right]=\alpha, Y_{+}^{i} \leq \theta_{2}: \\
P\left(\nu_{+}^{i}+\tau-\Delta \delta_{+}^{i}>\theta_{2} \mid \nu_{+}^{i}+\tau \leq \theta_{2},\right. \\
\left.\Delta \delta_{+}^{i}<0, Y_{+}^{i} \leq \theta_{2}\right) .
\end{gathered}
$$

For the first case, we have

$$
\begin{gathered}
\lim _{\Delta \theta_{1} \rightarrow 0} \frac{P\left(\nu_{-}^{i}+\tau-\Delta \delta_{-}^{i} \leq \theta_{2} \mid \nu_{-}^{i}+\tau>\theta_{2}, \Delta \delta_{-}^{i}>0\right)}{\Delta \theta_{1}} \\
=\frac{f\left(\nu_{*}^{i}+\theta_{2}-\nu_{-}^{i}\right)}{F\left(\nu_{*}^{i}+\eta_{i}\right)-F\left(\nu_{*}^{i}+\theta_{2}-\nu_{-}^{i}\right)} \frac{d \delta_{-}^{i}}{d \theta_{1}}
\end{gathered}
$$

if $\left(\xi_{i} \leq \theta_{2}-\nu_{-}^{i} \leq \eta_{i}, \frac{d \delta_{-}^{2}}{d \theta_{1}}<0\right)$ and 0 otherwise.

For the second case, we have given $Y_{+}^{i}>\theta_{2}$ :

$$
\begin{gathered}
\lim _{\Delta \theta_{1} \rightarrow 0} \frac{P\left(\nu_{+}^{i}+\tau-\Delta \delta_{+}^{i} \leq \theta_{2} \mid \nu_{+}^{i}+\tau>\theta_{2}, \Delta \delta_{+}^{i}<0\right)}{\Delta \theta_{1}} \\
=\frac{f\left(\nu_{*}^{i}+\theta_{2}-\nu_{+}^{i}\right)}{F\left(\nu_{*}^{i}+\eta_{i}\right)-F\left(\nu_{*}^{i}+\theta_{2}-\nu_{+}^{i}\right)}\left(-\frac{d \delta_{+}^{i}}{d \theta_{1}}\right)
\end{gathered}
$$

if $\left(\xi_{i} \leq \theta_{2}-\nu_{+}^{i} \leq \eta_{i}, \frac{d \delta_{+}^{i}}{d \theta_{1}}>0, Y_{+}^{i}>\theta_{2}\right) ; 0$ otherwise.

For the third case, we can further subdivide the cases based on $\mathbf{I}\left\{Y_{*}^{i}>\theta_{2}\right\}$, because when $Y_{*}^{i}>\theta_{2}$, the expected contribution due to the change turns out to be zero by symmetry. Thus, we can just consider the case $Y_{*}^{i} \leq \theta_{2}$, which must be added into the conditional distribution for $\tau_{i+1}$, yielding the conditional rate, given $Y_{+}^{i}>\theta_{2}, Y_{*}^{i} \leq \theta_{2}$ :

$$
\begin{gathered}
\lim _{\Delta \theta_{1} \rightarrow 0} \frac{P\left(\nu_{+}^{i}+\tau-\Delta \delta_{+}^{i} \leq \theta_{2} \mid \nu_{+}^{i}+\tau>\theta_{2}, \Delta \delta_{+}^{i}<0\right)}{\Delta \theta_{1}} \\
=\frac{f\left(\nu_{*}^{i}+\theta_{2}-\nu_{+}^{i}\right)}{F\left(\nu_{*}^{i}+\eta_{i}\right)-F\left(\nu_{*}^{i}+\theta_{2}-\nu_{+}^{i}\right)}\left(-\frac{d \delta_{+}^{i}}{d \theta_{1}}\right)
\end{gathered}
$$


if $\left(\xi_{i} \leq \theta_{2}-\nu_{+}^{i} \leq \eta_{i}, \frac{d \delta_{+}^{i}}{d \theta_{1}}>0, Y_{+}^{i}>\theta_{2}, Y_{*}^{i} \leq \theta_{2}\right)$;

0 otherwise.

For the fourth case, we again can further subdivide the cases based on $\mathbf{I}\left\{Y_{*}^{i}>\theta_{2}\right\}$, because this time when $Y_{*}^{i} \leq \theta_{2}$, the expected contribution due to the change turns out to be zero by symmetry. Thus, we can just consider the case $Y_{*}^{i}>\theta_{2}$, which must be added into the conditional distribution for $\tau_{i+1}$, yielding the conditional rate, given $Y_{+}^{i} \leq \theta_{2}, Y_{*}^{i}>\theta_{2}$ :

$$
\begin{gathered}
\lim _{\Delta \theta_{1} \rightarrow 0} \frac{P\left(\nu_{+}^{i}+\tau-\Delta \delta_{+}^{i} \leq \theta_{2} \mid \nu_{+}^{i}+\tau>\theta_{2}, \Delta \delta_{+}^{i}<0\right)}{\Delta \theta_{1}} \\
=\frac{f\left(\nu_{*}^{i}+\theta_{2}-\nu_{+}^{i}\right)}{F\left(\nu_{*}^{i}+\eta_{i}\right)-F\left(\nu_{*}^{i}+\theta_{2}-\nu_{+}^{i}\right)}\left(-\frac{d \delta_{+}^{i}}{d \theta_{1}}\right)
\end{gathered}
$$

if $\left(\xi_{i} \leq \theta_{2}-\nu_{+}^{i} \leq \eta_{i}, \frac{d \delta_{+}^{i}}{d \theta_{1}}>0, Y_{+}^{i} \leq \theta_{2}, Y_{*}^{i}>\theta_{2}\right)$; 0 otherwise.

Having completed the derivation of the event exchange rate term in Equation (1), we now turn to the change in the performance measure due to the event exchange. We need to construct two sample paths which represent the limit in which the exchange occurs, one in which the events "just miss" exchanging and the other in which the events "just make" exchanging. The paths are identical to the nominal (original) path up to the $i$ th replacement epoch with the following changes at the $(i+1)$ st replacement epoch (where we represent the newly constructed paths with a tilde representation), corresponding to each of the four previous cases:

$$
\begin{aligned}
\text { Case 1. } \tilde{\nu}_{j}^{i+1} & =\nu_{j}^{i}+\xi_{j}^{i}, \text { if } e_{j}^{i+1}=\phi, \\
\tilde{\nu}_{j}^{i+1} & =0, \text { if } j=* \text { or } e_{j}^{i+1}=\gamma, j \neq-, \\
P P 1: \tilde{\nu}_{-}^{i+1} & =\theta_{2}, \\
D N P 1: \bar{\nu}_{-}^{i+1} & =0 \\
g_{i+1}^{P P 1}-g_{i+1}^{D N P 1} & =-c_{R} ;
\end{aligned}
$$

Case 2. $\tilde{\nu}_{j}^{i+1}=\nu_{j}^{i}+\eta_{j}^{i}$, if $e_{j}^{i+1}=\phi, j \neq+$

$$
\tilde{\nu}_{j}^{i+1}=0, \text { if } j=* \text { or } e_{j}^{i+1}=\gamma,
$$$$
P P 2: \tilde{\nu}_{+}^{i+1}=0 \text {, }
$$$$
D N P 2: \tilde{\nu}_{+}^{i+1}=\theta_{2} \text {, }
$$$$
g_{i+1}^{P P 2}-g_{i+1}^{D N P 2}=+c_{R} \text {; }
$$

$\underline{\text { Case 3. }} \tilde{\nu}_{j}^{i+1}=\nu_{j}^{i}+\eta_{j}^{i}, \quad$ if $e_{j}^{i+1}=\phi, j \neq+$,

$$
\tilde{\nu}_{j}^{i+1}=0, \text { if } j=* \text { or } e_{j}^{i+1}=\gamma,
$$

PP3: $\tilde{\nu}_{+}^{i+1}=0$,

$D N P 3: \tilde{\nu}_{+}^{i+1}=0$, $g_{i+1}^{P P 3}-g_{i+1}^{D N P 3}=-c_{R}$;

$$
\begin{aligned}
\text { Case 4. } \tilde{\nu}_{j}^{i+1} & =\nu_{j}^{i}+\eta_{j}^{i}, \quad \text { if } e_{j}^{i+1}=\phi, j \neq+, \\
\tilde{\nu}_{j}^{i+1} & =0, \text { if } j=* \text { or } e_{j}^{i+1}=\gamma, \\
P P 4: \tilde{\nu}_{+}^{i+1} & =0 \\
D N P 4: \tilde{\nu}_{+}^{i+1} & =0 \\
g_{i+1}^{P P 4}-g_{i+1}^{D N P 4} & =+c_{R} ;
\end{aligned}
$$

Case 5 is identical to Case 1 . We note further that for Cases 3 and 4, no additional work need be done, because other than the cost difference at $t_{i+1}$, the two paths $P P$ and $D N P$ are identical; finally, Case 2 is similar to the negative of Case 1 , except that the upper bound $\eta_{i}$ is used instead of the lower bound $\xi_{i}$.

Defining the indicator function to be 0 if the index "-" does not exist, our final estimators are given by the following:

$$
\begin{aligned}
& \frac{d \bar{L}_{n}}{d \theta_{1}}=\sum_{i=1}^{n} \mathbf{I}\left\{\xi_{i} \leq \theta_{2}-\nu_{-}^{i} \leq \eta_{i}, \frac{d \delta_{-}^{i}}{d \theta_{1}}>0\right\} \\
& \times \frac{f\left(\nu_{*}^{i}+\theta_{2}-\nu_{-}^{i}\right)}{F\left(\nu_{*}^{i}+\eta_{i}\right)-F\left(\nu_{*}^{i}+\theta_{2}-\nu_{-}^{i}\right)} \frac{d \delta_{-}^{i}}{d \theta_{1}}\left(\bar{L}_{n}^{P P 1(i)}-\bar{L}_{n}^{D N P 1(i)}\right) \\
& -\sum_{i=1}^{n} \mathbf{I}\left\{\xi_{i} \leq \theta_{2}-\nu_{+}^{i} \leq \eta_{i}, \frac{d \delta_{+}^{i}}{d \theta_{1}}<0, Y_{+}^{i}>\theta_{2}, e_{+}^{i+1}=\phi\right\} \\
& \times \frac{f\left(\nu_{*}^{i}+\theta_{2}-\nu_{+}^{i}\right)}{F\left(\nu_{*}^{i}+\eta_{i}\right)-F\left(\nu_{*}^{i}+\theta_{2}-\nu_{+}^{i}\right)}\left(\frac{d \delta_{+}^{i}}{d \theta_{1}}\right)\left(\bar{L}_{n}^{P P 2(i)}-\bar{L}_{n}^{D N P 2(i)}\right) \\
& +\sum_{i=1}^{n} \mathbf{I}\left\{\xi_{i} \leq \theta_{2}-\nu_{+}^{i} \leq \eta_{i}, \frac{d \delta_{+}^{i}}{d \theta_{1}}<0, Y_{+}^{i}>\theta_{2}, e_{+}^{i+1}=\gamma, Y_{*}^{i} \leq \theta_{2}\right\} \\
& \quad \times \frac{f\left(\nu_{*}^{i}+\theta_{2}-\nu_{+}^{i}\right)}{F\left(\nu_{*}^{i}+\eta_{i}\right)-F\left(\nu_{*}^{i}+\theta_{2}-\nu_{+}^{i}\right)}\left(\frac{d \delta_{+}^{i}}{d \theta_{1}}\right) \frac{c_{R}}{n}
\end{aligned}
$$

$$
\begin{gathered}
-\sum_{i=1}^{n} \mathbf{I}\left\{\xi_{i} \leq \theta_{2}-\nu_{+}^{i} \leq \eta_{i}, \frac{d \delta_{+}^{i}}{d \theta_{1}}<0, Y_{+}^{-i} \leq \theta_{2}, Y_{*}^{-i}>\theta_{2}\right\} \\
\quad \times \frac{f\left(\nu_{*}^{i}+\theta_{2}-\nu_{+}^{i}\right)}{F\left(\nu_{*}^{i}+\eta_{i}\right)-F\left(\nu_{*}^{i}+\theta_{2}-\nu_{+}^{i}\right)}\left(\frac{d \delta_{+}^{i}}{d \theta_{1}}\right) \frac{c_{R}}{n},
\end{gathered}
$$

$$
\frac{d \bar{L}_{n}}{d \theta_{2}}=\sum_{i=1}^{n} \mathbf{I}\left\{\xi_{i} \leq \theta_{2}-\nu_{-}^{i} \leq \eta_{i}\right\}
$$$$
\times \frac{f\left(\nu_{*}^{i}+\theta_{2}-\nu_{-}^{i}\right)}{F\left(\nu_{*}^{i}+\eta_{i}\right)-F\left(\nu_{*}^{i}+\theta_{2}-\nu_{-}^{i}\right)}\left(\bar{L}_{n}^{P P 1(i)}-\bar{L}_{n}^{D N P 1(i)}\right) .
$$ 


\section{SPECIAL CASES}

The obvious way to estimate $\left(\bar{L}_{n}^{P P(i)}-\bar{L}_{n}^{D N P(i)}\right)$ is to simulate from the $(i+1)$ st replacement epoch to the $n$th replacement epoch for each of the two paths, take the difference, and add the appropriate $\left(g_{i+1}^{P P}-g_{i+1}^{D N P}\right)$ term. It is clear that this could potentially entail a substantial number of sub-simulations, and probably not computationally practical. For the cases of $N=2$ and $\theta_{2}=0$, we can derive efficient estimators, which we discuss in this section.

If $\theta_{2}=0$, then all components would always be simultaneously replaced, so in essence the system acts as a single component with the distribution being the minimum of all service times. It should be clear that for our performance measure the derivative with respect to $\theta_{1}$ is 0 , and our estimator will also give this, since every replacement epoch is a regenerative point and hence $d \delta_{j}^{i} / d \theta_{1}=0$ always. Although the derivative with respect to $\theta_{2}$ is non-zero, since every replacement epoch is regenerative, the quantity $\left(\bar{L}_{n}^{P P(i)}-\bar{L}_{n}^{D N P(i)}\right)$ always corresponds to a regenerative starting point, and hence can be very efficiently estimated using the sample path itself without need for additional simulation.

For $\theta_{2} \neq 0$, we also have simplification for $N=2$ and large $n$, because the term $\left(\bar{L}_{n}^{P P(i)}-\bar{L}_{n}^{D N P(i)}\right)$ will no longer depend on various ages at $t_{i}$, simply because there won't be any other components besides the two involved in the potential event exchange.

\section{Case 1:}

$\tilde{\nu}_{*}^{i+1}=0, \quad P P 1: \quad \tilde{\nu}_{-}^{i+1}=\theta_{2}, \quad D N P 1: \tilde{\nu}_{-}^{i+1}=0 ;$

Case 2:

$\tilde{\nu}_{*}^{i+1}=0, \quad P P 1: \quad \tilde{\nu}_{+}^{i+1}=0, \quad D N P 1: \tilde{\nu}_{+}^{i+1}=\theta_{2} ;$

i.e, taking " $*$ " as component $1, D N P 1$ is the path starting with ages $(0,0)$ (regenerative point), whereas $P P 1$ is the path starting with ages $\left(0, \theta_{2}\right)$, and $D N P 2$ and $P P 2$ simply correspond to the reverse of this. Recall that if the "-" needed for the first and fourth cases are missing, then there is no contribution for that replacement epoch. When there are only two components, the "-" component - if it exists - will correspond to the " + " component, which is simply the component other than the "*" component, and we also have $\xi_{i}=\theta_{2}-\nu_{i}^{i}, \quad \eta_{i}=Y_{-}^{i}-\nu_{i}^{i}$. In addition, the first condition in the indicator functions will automatically be satisfied. These results simplify the estimators given by Equations (2) and (3).

Furthermore, for large $n$, we get the following simplification by using the construction for $D N P$ and

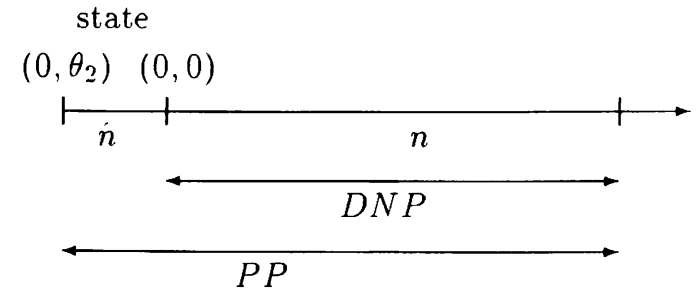

Figure 3: Sample Path Used to Construct $D N P$ and $P P$

$P P$ depicted in Figure 3 (Case 1):

$$
\begin{aligned}
\bar{L}^{P P}-\bar{L}^{D N P} & \approx \bar{L}_{n+\dot{n}}^{P P}-\bar{L}_{n}^{D N P} \\
& =\frac{1}{n+\dot{n}} \sum_{i=1}^{n+\dot{n}} g_{i}-\frac{1}{n} \sum_{i=\hat{n}}^{\dot{n}+n} g_{i} \\
& =\frac{1}{n+\dot{n}}\left(\sum_{i=1}^{n} g_{i}+\sum_{i=\hat{n}}^{n} g_{i}\right)-\frac{1}{n} \sum_{i=\hat{n}}^{n+n} g_{i} \\
& =\frac{1}{n+\dot{n}} \sum_{i=1}^{n} g_{i}-\frac{\hat{n}}{n(n+\dot{n})} \sum_{i=\hat{n}}^{n+n} g_{i} \\
& \approx \frac{1}{n+\dot{n}}\left(\sum_{i=1}^{\dot{n}} g_{i}-\dot{n} \bar{L}_{n}\right) .
\end{aligned}
$$

Because this quantity is independent of $i$, it can be taken outside the summation in the expressions for the estimators, and estimated completely independently (e.g.. "off-line") from the rest of the estimator, where $\dot{n}$ and $\sum_{i=1}^{\dot{n}} g_{i}$ are the quantities which need to be estimated. They can be efficiently estimated by simply running one additional (relatively very short) simulation which starts from the initial condition with ages $\left(0, \theta_{2}\right)$ and ends when it first hits the regenerative point of both components being replaced. Of course, to get better estimates, this can be done for multiple replications.

Example: For illustrative purposes, we now consider an example for the $N=2$ case to see the resulting simplifications from the preceding analysis. If the components have uniformly distributed lifetimes, with $\theta_{1}$ as the upper bound, then we have the following for the hazard-rate-like functions that appear in the estimators:

$\frac{f\left(\nu_{*}^{i}+\theta_{2}-\nu_{+}^{i}\right)}{F\left(\nu_{*}^{i}+\eta_{i}\right)-F\left(\nu_{*}^{i}+\theta_{2}-\nu_{+}^{i}\right)}=\frac{1}{\min \left(Y_{+}^{i}, \theta_{1}-\nu_{*}^{i}+\nu_{+}^{i}\right)-\theta_{2}}$

(recall that $Y_{-}^{i}=Y_{+}^{i}$, if "-" exists, where "+" will correspond to the component other than "*"), and the estimators given by Equations (2) and (3) become the following easy to implement and computationally efficient estimators: 


$$
\begin{aligned}
& \frac{d \bar{L}_{n}}{d \theta_{1}}=\left(\bar{L}^{P P}-\bar{L}^{D N P}-c_{R} / n\right) \times \\
& {\left[\sum_{i=1}^{n} \mathbf{I}\left\{\frac{d \delta_{-}^{i}}{d \theta_{1}}>0\right\} \frac{1}{\tilde{Y}_{+}^{i}-\theta_{2}} \frac{d \delta_{-}^{i}}{d \theta_{1}}\right.} \\
& \left.\quad+\mathbf{I}\left\{\frac{d \delta_{+}^{i}}{d \theta_{1}}<0, Y_{+}^{i}>\theta_{2}, e_{+}^{i+1}=\phi\right\} \frac{1}{\tilde{Y}_{+}^{i}-\theta_{2}} \frac{d \delta_{+}^{i}}{d \theta_{1}}\right] \\
& +\sum_{i=1}^{n}\left[\mathbf{I}\left\{\frac{d \delta_{+}^{i}}{d \theta_{1}}<0, Y_{+}^{i}>\theta_{2}, e_{+}^{i+1}=\gamma, Y_{*}^{i} \leq \theta_{2}\right\}\right. \\
& \left.\quad-\mathbf{I}\left\{\frac{d \delta_{+}^{i}}{d \theta_{1}}<0, Y_{+}^{i} \leq \theta_{2}, Y_{*}^{i}>\theta_{2}\right\}\right] \frac{1}{\tilde{Y}_{+}^{i}-\theta_{2}} \frac{d \delta_{+}^{i}}{d \theta_{1}} \frac{c_{R}}{n}, \\
& \frac{d \bar{L}_{n}}{d \theta_{2}}=\left(\bar{L}^{P P}-\bar{L}^{D N P}-c_{R} / n\right) \sum_{i=1}^{n} \frac{\mathbf{I}\left\{e_{j}^{i+1}=\gamma, j \neq *\right\}}{\tilde{Y}_{+}^{i}-\theta_{2}},
\end{aligned}
$$

where $\tilde{Y}_{+}^{i}=\min \left(Y_{+}^{i}, \theta_{1}-\nu_{*}^{i}+\nu_{+}^{i}\right)$ and $\left(\bar{L}^{P P}-\bar{L}^{D N P}\right)$ is given by Equation (4).

\section{CONCLUDING REMARKS}

Threshold parameters arise frequently in the optimization of discrete-event systems, so the availability of gradient estimates is an important area of research. As noted in the introduction, perturbation analysis is a gradient estimation technique which has been applied to a wide variety of these types of problems. We have shown that it is possible to derive gradient estimates for replacement problems in maintenance theory; however, the estimators we have derived here do not appear to be computationally efficient except for the special cases $N=2$ or $\theta_{2}=0$, which are of limited practical use. The estimation of $\left(\bar{L}_{n}^{P P(i)}-\bar{L}_{n}^{D N P(i)}\right)$ requires in general on the order of one separate subsimulation for each replacement epoch, so there might be on the order of $n$ extra sub-simulations required per replication, though many of them would be quite short. For the special cases, this extra simulation is eliminated. An alternative approach which might be more successful for this problem is the standard clock approach (see, e.g., Vakili 1991). Proofs for unbiasedness of the gradient estimators derived here have been omitted, but as is usual for perturbation analysis estimators, the key tool would be the dominated convergence theorem, which allows the interchange of derivative (limit) and expectation (integral); such a proof would proceed along lines similar to the ones in Fu and Hu (1992), though as we noted already, this simple system does not satisfy some of the assumptions usually given for a GSMP framework.
Finally we note that gradient estimation techniques from the discrete-event simulation community need not be restricted to the "traditional" models of operations research such as queueing, inventory, and reliability. Another application to a threshold-like parameter is considered in $\mathrm{Fu}$ and $\mathrm{Hu}$ (1993), in which is derived an efficient unbiased estimator (in contrast to the problem studied here, requiring only one extra short simulation per replication) for the gradient of an American call option price with respect to its early exercise threshold at an ex-dividend point. It is then demonstrated how this gradient estimator can be used to do option pricing for American-style (where early exercise is possible) options, for which heretofore the finance community had not considered Monte Carlo simulation as a viable alternative.

\section{ACKNOWLEDGMENTS}

Michael Fu was supported in part by a General Research Board Award from the University of Maryland at College Park. Jian-Qiang $\mathrm{Hu}$ was supported in part by the National Science Foundation (NSF) under grants EID-9212122 and DDM-9212368. Leyuan Shi was supported in part by the Office of Naval Research (ONR) and the Defense Advanced Research Projects Agency (DARPA) under contract N0001491-C-0195 to Honeywell and the Computer Science Department of the University of Maryland at College Park.

\section{REFERENCES}

Caramanis, M. and Liberopolous, G. 1992. Perturbation Analysis for the Design of Flexible Manufacturing Flow Controllers, Operations Research 40: 1107-1126.

Fu, M.C. 1993. Sample Path Derivatives for (s,S) Inventory Systems. to appear in Operations Research.

Fu, M.C. 1994. Optimization Using Simulation: A Review. to appear in Annals of Operations Research.

Fu, M.C. and J.Q. Hu. 1992. Extensions and Generalizations of Smoothed Perturbation Analysis in a Generalized Semi-Markov Process Framework. IEEE Transactions on Automatic Control 37: $1483-1500$.

Fu, M.C. and J.Q. Hu. 1993. Sensitivity Analysis for Monte Carlo Simulation of Option Pricing. submitted for publication.

Glasserman, P. 1991. Gradient Estimation Via Perturbation Analysis. Kluwer Academic. 
Gong, W.B. 1988. Smoothed Perturbation Analysis Algorithm for a G/G/1 Routing Problem. Proceedings of the 1988 Winter Simulation Conference: 525-531.

Gong, W.B. and Y.C. Ho. 1987. Smoothed Perturbation Analysis of Discrete-Event Dynamic Systems. IEEE Transactions on Automatic Control 32: 858867.

Ho, Y.C. and X.R. Cao. 1991. Perturbation Analysis and Discrete Event Dynamic Systems. Kluwer Academic.

L'Ecuyer, P. 1990. A Unified View of the IPA, SF, and LR Gradient Estimation Techniques, Management Science 36: 1364-1383.

Rubinstein, R.Y. 1992. Sensitivity Analysis of Discrete Event Systems by the "Push Out" Method. Annals of Operations Research 39.

Vakili, P. 1991. Using a Standard Clock Technique for Efficient Simulation. Operations Research Letters 10: 445-452.

Wardi, Y., Zazanis, M.A., and Luo, M. 1991. Consistency of Perturbation Analysis for a Queue with Finite Buffer Space and Loss Policy. Journal of Optimization Theory and Applications 68: 181-202.

\section{AUTHOR BIOGRAPHIES}

MICHAEL C. FU is an Assistant Professor of Management Science \& Statistics in the College of Business and Management at the University of Maryland at College Park. He holds S.B. and S.M. degrees in electrical engineering and an S.B. degree in mathematics from MIT (1985) and a Ph.D. in applied mathematics from Harvard University (1989). His research interests include stochastic derivative estimation and stochastic optimization, particularly with applications towards manufacturing systems and inventory control. He teaches courses in applied probability, stochastic processes, discrete-event simulation, and operations management.

JIAN-QIANG HU is an Assistant Professor in the Department of Manufacturing Engineering at Boston University. He holds a B.S. degree in applied mathematics from Fudan University, China (1985), and M.S. and Ph.D. degrees in applied mathematics from Harvard University $(1987,1990)$. His research interests include modeling, sensitivity analysis, simulation, optimization, and control of discrete event stochastic systems and queueing networks with applications to manufacturing systems and communication networks. He was awarded the Honorable Mention by the Operations Research Society of America (ORSA) in the 1991 George E. Nicholson Student Paper Com- petition.

LEYUAN SHI is a Research Associate in the Institute for Advanced Computer Studies at the University of Maryland at College Park. She received a B.S. degree from Nanjing Normal University, China (1982) and a M.S. degree in applied mathematics from Tsinghua University, China (1985), and M.S. and Ph.D degrees in applied mathematics from Harvard University $(1990,1992)$. Her research interests include modeling, analysis and control of discrete event stochastic systems, particularly with the applications on design and control of computer networks, multicomputer systems and communication protocols. 